\title{
Curve-pleated Structures
}

\author{
CAIGUI JIANG, KAUST \\ KLARA MUNDILOVA, TU Wien \\ FLORIAN RIST, TU Wien, KAUST \\ JOHANNES WALLNER, TU Graz \\ HELMUT POTTMANN, KAUST
}

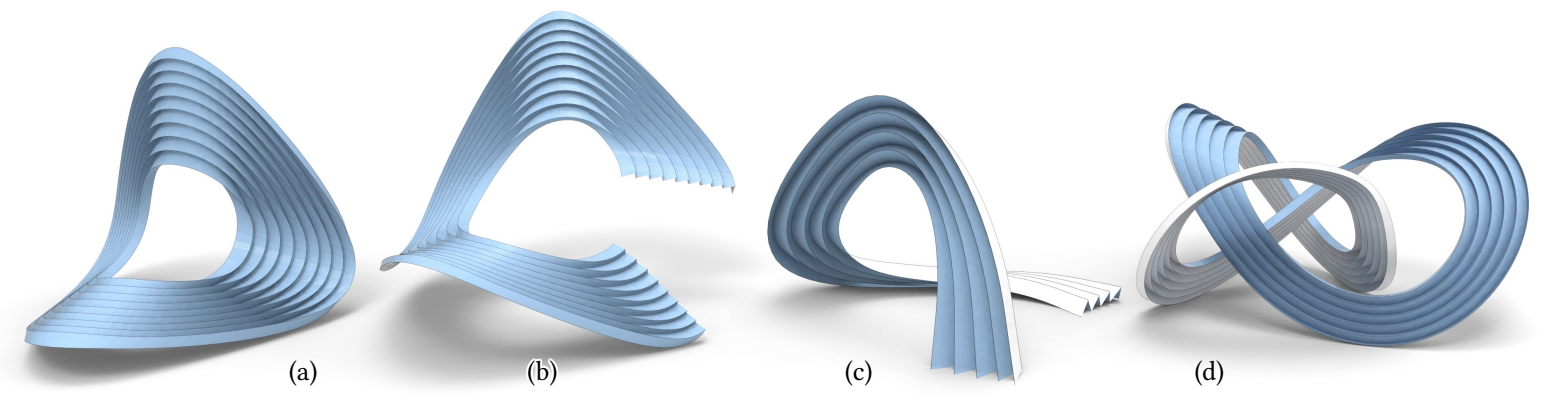

Fig. 1. Our work on pleated surfaces is motivated by discrete differential geometry and inspired by curved-folding art. These images show quad meshes with planar faces which approximate a surface with smooth curved creases, which are exactly developable, and which actually are conical meshes. As such, they constitute a structure-preserving discretization of a nice class of curved-folding surfaces, namely ones which enjoy flat-foldability, and constant fold angle. These properties apply to both the continuous and the discrete case. The two surfaces at left are connected by a ruling-preserving isometry.

In this paper we study pleated structures generated by folding paper along curved creases. We discuss their properties and the special case of principal pleated structures. A discrete version of pleated structures is particularly interesting because of the rich geometric properties of the principal case, where we are able to establish a series of analogies between the smooth and discrete situations, as well as several equivalent characterizations of the principal property. These include being a conical mesh, and being flatfoldable. This structure-preserving discretization is the basis of computation and design. We propose a new method for designing pleated structures and reconstructing reference shapes as pleated structures: we first gain an overview of possible crease patterns by establishing a connection to pseudogeodesics, and then initialize and optimize a quad mesh so as to become a discrete pleated structure. We conclude by showing applications in design and reconstruction, including cases with combinatorial singularities. Our work is relevant to fabrication in so far as the offset properties of principal pleated structures allow us to construct curved sculptures of finite thickness.

CCS Concepts: • Computing methodologies $\rightarrow$ Computer graphics; Shape modeling; Mesh geometry models.

Authors' addresses: Caigui Jiang, KAUST, caigui.j@gmail.com; Klara Mundilova, TU Wien, klara.mundilova@gmail.com; Florian Rist, TU Wien, KAUST, florian.rist@tuwien. ac.at; Johannes Wallner, TU Graz, j.wallner@tugraz.at; Helmut Pottmann, KAUST, helmut.pottmann@gmail.com.

Permission to make digital or hard copies of all or part of this work for personal or classroom use is granted without fee provided that copies are not made or distributed for profit or commercial advantage and that copies bear this notice and the full citation on the first page. Copyrights for components of this work owned by others than ACM must be honored. Abstracting with credit is permitted. To copy otherwise, or republish, to post on servers or to redistribute to lists, requires prior specific permission and/or a fee. Request permissions from permissions@acm.org.

(c) 2019 Association for Computing Machinery.

0730-0301/2019/11-ART169 \$15.00

https://doi.org/10.1145/3355089.3356540
Additional Key Words and Phrases: pleated surface, curved crease, developable surface, computational origami, computational fabrication, discrete differential geometry, conical mesh, pseudo-geodesic

\section{ACM Reference Format:}

Caigui Jiang, Klara Mundilova, Florian Rist, Johannes Wallner, and Helmut Pottmann. 2019. Curve-pleated Structures. ACM Trans. Graph. 38, 6, Article 169 (November 2019), 13 pages. https://doi.org/10.1145/3355089.3356540

\section{INTRODUCTION}

The creation of unexpected geometric shapes from flat sheets of paper is a fascinating topic and has been for centuries. Origami, which means folding paper along straight edges without cutting, has not only been a medium of art, but also the object of systematic study from the mathematical and computational viewpoint [Demaine and O'Rourke 2007]. In the present paper we extend the origami principle and generate geometric shapes by folding along curves. Our inspiration comes from artwork and from recent studies in structural properties and materials science, where small-scale folding patterns imposed on geometric shapes increasingly play a role.

Approximating a given surface by a curved-crease surface is a largely open and difficult problem. A solution would provide strong support in the computational design process. The currently available methods work in the reverse direction: One first creates the unfolded state and derives the spatial shape from that. The shape problem of curved-crease surfaces is global and of a different nature than for straight-edge origami. This is because the developable strips which make up the surface influence each other and usually deform simultaneously. 


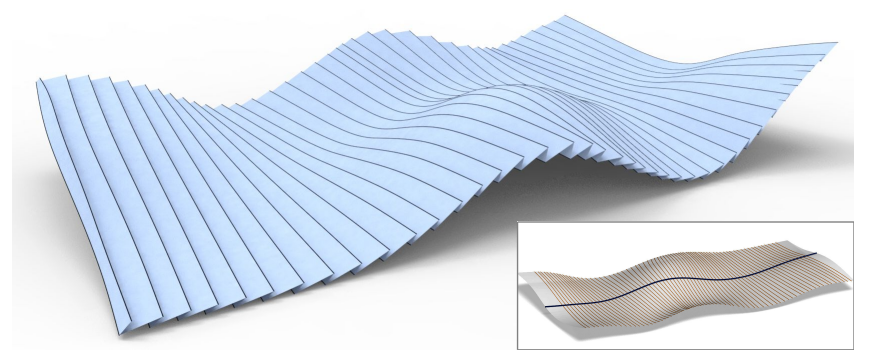

Fig. 2. Reconstructing the 'flying carpet' glass roof in the Louvre's Cour Visconti by a pleated structure. To solve this kind of reconstruction problem, folds are first initialized from a pattern of pseudo-geodesic curves (here: orthogonal to the blue guiding curve), and are then subjected to global optimization.

\subsection{Contributions}

In this work, we consider pleated structures, PLs for short, which exhibit zigzag folds along curves on a small scale, but which on a larger scale represent smooth geometric shapes. The system of mountain and valley folds corresponds to a system of curves on the reference shape. By establishing connections to differential geometry, we are able to approach design and reconstruction problems. In particular, our contributions are the following:

- We study the geometry of pleated structures and their discretization as quad meshes with planar faces. Transformations well known in discrete differential geometry help to explore the design space. In particular, we emphasize the reflected ruling case which is discretized as conical meshes. This connection between conical meshes and special "principal" pleated structures is new and puts the latter into well-established theories of discrete differential geometry. Such principal pleated structures (PPLS for short) possess constantdistance offsets and also enjoy a continuous isometric unfolding which preserves the rulings, in both the continuous and the discrete cases. In the terminology of discrete differential geometry, we have achieved an integrable discretization.

- We present an optimization algorithm for design and reconstruction of pleated structures. The main contribution here is the initialization, which works through special curves (pseudo-geodesics) on the reference surface, and through the construction of surfaces where suitable guiding curves have controlled distances. The use of pseudo-geodesics corresponds to the desire to create pleated structures where we control the inclination of folds w.r.t. the reference surface. Thus, for the first time, we are able to approximate general shapes by curved-folding surfaces.

- We illustrate applications, where properties of curved-creased structures benefit fabrication. The effectiveness of our algorithms is demonstrated by means of examples, most of which could not be achieved with the current state of the art in the digital design of pleated structures. This applies in particular to flexible principal pleated structures.

\subsection{Previous Work}

The most influential early work on the design of curved-crease surfaces is by David Huffman [1976]. He collects geometric properties, in particular the equal-angle condition involving the osculating plane of crease curves, and then uses his theoretical insights for the design of origami with both straight and curved creases, see [Demaine et al. 2011a], [Davis et al. 2013] and [Koschitz 2014]. Differential-geometric properties of curved folds were collected by [Fuchs and Tabachnikov 1999; Kergosien et al. 1994] from the viewpoint of Mathematics and Computer Science, respectively. Recent contributions to the differential geometry of curved folds include [Demaine et al. 2015a, 2018]. The latter discuss the important reflection ruling case in depth. Without knowing his work would be relevant for curved-crease surfaces, pseudo-geodesics on special surfaces were discussed by [Wunderlich 1950a,b,c,d].

Surfaces with curved creases have many connections to ones where creases are straight, not least because of discretization. Particularly interesting problems for straight crease surfaces are the ones relevant also in the curved crease case.

One type of result regards the realizability of specific geometric shapes, such as the nonexistence of the 'pleated hypar' folding [Demaine et al. 2011b], or the wrappability of any polyhedron by a strip [Demaine et al. 2000]. The 'Origamizer', see [Demaine and Tachi 2017; Tachi 2010b] is an algorithm for computing a crease pattern for any given polyhedron.

Relevant to the discretization employed in the present paper are rigidly foldable quad meshes, where rigid faces are connected with hinges in the edges. They are not rigid but possess a 1-parameter continuous flexion. Early work by Kokotsakis [1933] is being continued by [Tachi 2009, 2013; Tachi and Epps 2011] and others. [Schief et al. 2008] established a connection between rigid foldability and integrable systems. More recently, Evans et al. [2015a; 2015b] found further conditions in terms of angle multipliers for a fold pattern to be rigidly foldable. Contributions to the more general topic of mechanisms based on folded surfaces include [Kilian et al. 2017]. Chen et al. [2015] consider an important practical aspect, namely structures of nonzero thickness.

There have been different computational approaches to curved crease surfaces. Specific special classes of pleated structures are those with rotational symmetry [Mitani 2009a], or designs by iteration of reflection, see [Mitani 2012; Mitani and Igarashi 2011] for the 2D case and [Röschel 2019] for a spatial version. The survey paper [Demaine et al. 2015b] reviews masterpieces of curved-crease folding and the corresponding design methods. The parametric design of origami surfaces with periodic tessellations is the topic of [Gardiner et al. 2018]. Analysis together with design has been done by Demaine et al. [2015a; 2018] for 'lens' crease patterns, and crease patterns formed by conics.

Kilian et al. [2008] used developable quad meshes for digital reconstruction of general curved-crease surfaces. As to modeling, Frey [2004] studied buckled developables by means of triangle meshes. Generally, methods for modeling developables may include developables with creases, e.g. [Tang et al. 2016], where developability is imposed on spline surfaces for geometric design, or [Rabinovich et al. 2018a,b], where developables resp. their deformations are modelled via discrete nets of orthogonal geodesics resp. appropriate flows of such nets. Both treat creases in the way of two developables to either side of the crease and impose a flattenability condition along it. Solomon et al. [2012] perform modeling with triangle meshes 
constrained by developability. Another mesh-based approach to modeling of general developables (without focus on curved creases) is proposed by Stein et al. [2018]. The fast GPU solver for origami and its generalizations by Ghassaei et al. [2018] also handles discrete curved creases.

A fundamental question is the geometric interaction between small-scale crease patterns on the one hand, and global properties of geometric shapes on the other. This is studied e.g. by [Dudte et al. 2016]. Song et al. [2017] analyzes curved-crease patterns which simultaneously fit two design surfaces of common rotational symmetry.

There have been many contributions about material properties of creases, including curved creases. [Ben Amar and Pomeau 1997] investigate the scars left after crumpling, and propose to extend the class of $C^{2}$ smooth developables so that all spatial closed curves can occur as boundary of a developable. [Cerda and Mahadevan 1998] and [Cerda et al. 1999] discuss crescent-like singularities resp. 'conical dislocations' in elastic sheets. Barois et al. [2014] considers nondevelopable thin sheets under high curvature. Repetitive patterns are analyzed by [Wei et al. 2013]. For structural properties of curved-crease surfaces we point to [Lebée 2015] and [Raducanu et al. 2016].

As to the shapes assumed by surfaces after a curved crease is imposed, [Dias et al. 2012], [Dias and Santangelo 2012] model the shape of an annulus with concentric creases (see also [Mouthuy et al. 2012]). A more general theory is developed by Dias and Audoly [2014; 2015], where the shape assumed by curved-crease surfaces is interpreted as a certain buckling mode. The connection between buckling and curved creases also works in reverse: Lee et al. [2019] propose embedded small curved-crease structures to guide the failure mode in a certain direction. Self-folding materials are analyzed by [Miyashita et al. 2015].

A very interesting line of research is materials whose properties are defined by small-scale patterns. [Seffen 2012] discusses in a systematic manner the properties of structures with local, discrete corrugations. Curved-crease patterns occurring naturally in crumpled leaves are proposed as basis of carbon fiber reinforced

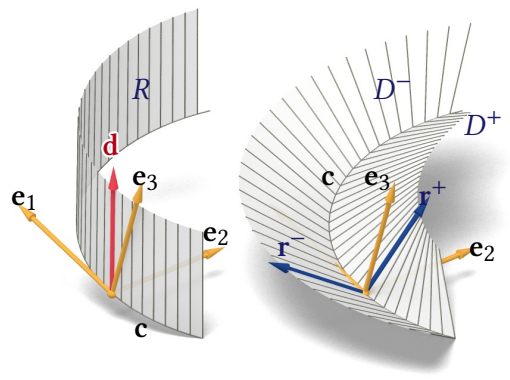

c............. crease curve $\mathbf{e}_{1} \ldots \ldots \ldots$ unit tangent vector $\mathbf{e}_{2} \ldots \ldots$ principal normal vector $\mathbf{e}_{3} \ldots \ldots \ldots$. binormal vector $\mathbf{d}=\tau \mathbf{e}_{1}+\kappa \mathbf{e}_{3}$. Darboux vector $\mathbf{r}^{+}, \mathbf{r}^{-} \ldots \ldots \ldots \ldots$. rulings $D^{+}, D^{-} \ldots \ldots \ldots$ developables $R$...... rectifying developable

Fig. 3. The moving Frenet-Serret frame $\mathbf{e}_{1}, \mathbf{e}_{2}, \mathbf{e}_{3}$ of a curve $\mathbf{c}$ is used to locally express developable surfaces which contain c. Left: With $\kappa, \tau$ as curvature and torsion, the vector $\tau \mathbf{e}_{1}+\kappa \mathbf{e}_{3}$ defines the rulings of the rectifying developable $R$ (whose normal vector is $\mathbf{e}_{2}$, and whose development straightens c). Right: If $\mathbf{c}$ is a curved fold in a surface $D^{+} \cup D^{-}, R$ bisects the developables $D^{+}, D^{-}$. This symmetry applies to tangent planes; in general, rulings $\mathbf{r}^{+}, \mathbf{r}^{-}$are not symmetric w.r.t. the plane $\left[\mathbf{e}_{1}, \mathbf{e}_{3}\right]$. composite foldcores by $\mathrm{Du}$ et al. [2019]. The elastic response of curved-crease foldcores is studied by [Gattas and You 2015]. Smallscale structures not confined to creases but involving also cutting are in general discussed by [Sussman et al. 2015] and [Callens and Zadpoor 2018]. We also point to the creation of auxetic materials by this principle, by Konaković et al. [2016; 2018].

\section{GEOMETRY OF CURVED FOLDS}

In this paper we study pleated structures, PLs for short. This means surfaces which are smooth except for crease curves, and which are isometrically developable onto a 2D domain. This developability is required to hold locally, not globally - we allow surfaces which must be cut along curves before they can be unfolded.

\subsection{Basic differential geometry of curved folds}

We start our discussion with a few well-known facts about a crease curve $\mathbf{c}$ contained in a piecewise smooth surface which can be isometrically mapped to a flat 2D domain, see e.g. [Fuchs and Tabachnikov 1999]. The notation we use is defined in Fig 3, cf. [do Carmo 1976]. The crease is contained in a unique developable $R$ which upon unfolding maps $\mathbf{c}$ to a straight line. The surface under consideration is the union of two developables $D^{+}, D^{-}$to either side of the crease which lie symmetric to $R$. The normal vector of $R$ is the crease curve's principal normal vector $\mathbf{e}_{2}$, while the normal vectors of $D^{+}, D^{-}$are represented by

$$
\mathbf{n}^{+}=\cos u \mathbf{e}_{2}+\sin u \mathbf{e}_{3}, \quad \mathbf{n}^{-}=\cos (\pi-u) \mathbf{e}_{2}+\sin (\pi-u) \mathbf{e}_{3},
$$

where $2 u=\phi$ is the angle of the crease. Assuming an arc length parametrization $\mathbf{c}(s)$ of the crease, we compute the rulings of $D^{+}$as $\mathbf{r}^{+}=\mathbf{n}^{+} \times \frac{d}{d s} \mathbf{n}^{+}$and analogous for $D^{-}$. For that we use the equations of the moving frame, $\frac{d}{d s} \mathbf{e}_{1}=\kappa \mathbf{e}_{2}, \frac{d}{d s} \mathbf{e}_{2}=-\kappa \mathbf{e}_{1}+\tau \mathbf{e}_{3}, \frac{d}{d s} \mathbf{e}_{3}=-\tau \mathbf{e}_{2}$, with $\kappa, \tau$ as curvature and torsion. We get

$$
\begin{aligned}
\frac{d}{d s} \mathbf{n}^{+} & =-\kappa \cos u \mathbf{e}_{1}+\left(\frac{d u}{d s}+\tau\right)\left(-\sin u \mathbf{e}_{2}+\cos u \mathbf{e}_{3}\right), \\
\mathbf{r}^{+} & =\left(\frac{d u}{d s}+\tau\right) \mathbf{e}_{1}-\kappa \sin u \cos u \mathbf{e}_{2}+\kappa \cos ^{2} u \mathbf{e}_{3} .
\end{aligned}
$$

Replacing $u$ by $\pi-u$ yields the ruling vector $\mathbf{r}^{-}$. If we let $u=0$, we retrieve the rulings of the rectifying developable $R$; they are given by the Darboux vector $\mathbf{d}=\tau \mathbf{e}_{1}+\kappa \mathbf{e}_{3}$.

\subsection{Principal pleated structures (continuous case)}

Creases with a constant fold angle have a particularly interesting geometry, which also turns out to be practically very useful. Equation (1) shows that in this case,

$$
\mathbf{r}^{ \pm}=\tau \mathbf{e}_{1} \mp \frac{1}{2} \kappa \sin \phi \mathbf{e}_{2}+\frac{1}{2} \kappa(1+\cos \phi) \mathbf{e}_{3},
$$

with $\phi=2 u$ as opening angle of the crease. The rulings are symmetric w.r.t. reflection in $\left[\mathbf{e}_{1}, \mathbf{e}_{3}\right]$. It is elementary to verify that the Darboux vector $\mathbf{d}=\tau \mathbf{e}_{1}+\kappa \mathbf{e}_{3}$, when projected onto the tangent planes of $D^{+}, D^{-}$, results in the rulings. We therefore have a cone whose axis is $\mathbf{d}$ and which touches $D^{+}, D^{-}$along the rulings, see Fig. 4. The symmetric position of rulings also implies that they form the same angle with the crease, and we have the reflection-ruling case studied by [Demaine et al. 2018]. These geometric facts will be the basis of discretization. 


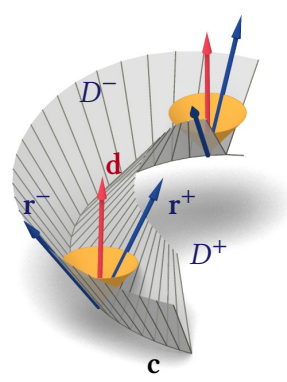

Fig. 4. A 'principal' crease. We observe the constant fold angle of the crease and the circular cone moving along the crease (in a non-rigid way). Its axis is the Darboux vector $\tau \mathbf{e}_{1}+\kappa \mathbf{e}_{3}$ of the crease curve, and it touches developables $D^{+}, D^{-}$along the rulings.

Connection to discrete differential geometry. If a quad mesh with planar faces approximates a smooth surface, existence of a tangent cone in each vertex means that this mesh follows principal curvature lines [Bobenko and Suris 2009; Liu et al. 2006]. Similarly, if a piecewise smooth surface composed of developable strips approximates a smooth surface, and there is a tangent cone in each vertex, then the strips follow principal curvature lines [Pottmann et al. 2008]. This is exactly the situation described above and by Fig. 4 except for the fact that our pleated structures approximate smooth surfaces only in a zigzag manner and we cannot infer any connection to principal curvature lines. Neither can we use the cone axes as surface normals.

We nevertheless define a principal pleated structure, or PPLs for short, as a pleated structure where the fold angle is constant along each crease. All properties of semidiscrete conical surfaces shown e.g. by [Pottmann et al. 2008] apply, such as existence of offsets at constant distance.

Remark on Darboux transforms. There are even more relations to differential geometry. For any PLs, the normal vectors of its developable pieces form a Gauss image consisting of curves. A look at the Gauss image curves $\mathbf{n}^{+}, \mathbf{n}^{-}$to both sides of a crease reveals firstly that the binormal $\mathbf{e}_{3}$ of the crease is the spherical midpoint of $\mathbf{n}^{+}, \mathbf{n}^{-}$. The equation $\frac{d}{d s} \mathbf{e}_{3}=-\tau \mathbf{e}_{2}$ shows that its derivative is parallel to $\mathbf{n}^{+}-\mathbf{n}^{-}$. The curves $\mathbf{n}^{+}, \mathbf{n}^{-}$thus form a spherical Darboux transform pair. The known complexity of Darboux transforms mirrors the known difficulties in creating pleated structures.

Continuous unfolding. Principal pleated structures have a remarkable property, discussed by [Demaine et al. 2018] in the smooth

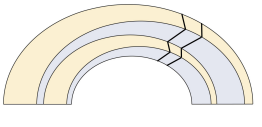
case and by [Tachi 2009] in a discrete version: There is a continuous isometric unfolding in which the rulings of the developable strips are preserved. This is not the case for general PLs. Fig. 5 shows an example. The corresponding unfolded state with rulings is shown in the inset figure. One can observe the reflection-ruling property. We return to this topic when discussing discrete pleated structures.

Relation to pseudo-geodesics. It is interesting that the so-called pseudo-geodesic curves on surfaces occur multiple times in the context of curved-folding structures. They are defined by the property that their osculating planes have constant angle with the reference surface (equivalently such a constant angle requirement may be imposed on the binormal vector or on the rectifying developable). The cases of $90^{\circ}$ and $0^{\circ}$ angle correspond to geodesics and asymptotic curves, respectively [do Carmo 1976]. We will later use pseudo-

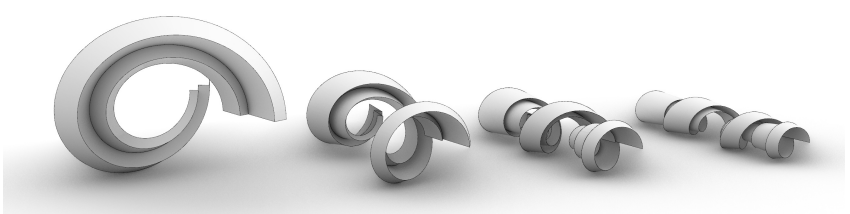

Fig. 5. Continuous flexion of a principal pleated structure. This example, whose crease curves in the unfolded state are ellipses, is analogous to the ones of [Mundilova 2019]. It demonstrates the general tendency of PLS to become rolled up as the crease angle decreases.

geodesics in our layout of crease curves, but there is a more direct connection: The constant angle condition in a PPLS expresses the fact that a crease $D^{+} \cap D^{-}$is a pseudo-geodesic of both $D^{+}$and $D^{-}$.

W. Wunderlich determined all PPLs composed of cylinders and cones, by studying curves occurring as pseudo-geodesics on cylinders and cones, and even on two such surfaces simultaneously [Wunderlich 1950a,b,c,d]. In an unfolded state, rulings have the property that they either are all parallel (cylinder case) or pass through a point (cone case). Together with the reflection-ruling property this implies that in the unfolded state, crease curves must be conic sections, see [Demaine et al. 2018].

\subsection{Discrete pleated structures}

For computing with pleated structures, we need a discretization. Fortunately, there is a natural discretization which exactly reproduces key properties of smooth PLS.

We use quad meshes with planar faces (in the same way as [Kilian et al. 2008]), where one family of edge polylines represents the crease curves, and the transverse edges represent rulings. A sequence of consecutive rulings forms a zigzag polyline. Developability is expressed by the requirement that in each vertex, the sum of incident angles equals 360 degrees, i.e.,

$$
\omega_{1}+\omega_{2}+\omega_{3}+\omega_{4}=2 \pi .
$$

It follows directly that the discrete pleated structures form a class of discrete surfaces largely invariant under mesh parallelism, resp. Combescure transforms [Bobenko and Suris 2009]. Assume two combinatorially equivalent quad meshes $(V, E, F)$ and $(\bar{V}, \bar{E}, \bar{F})$ such

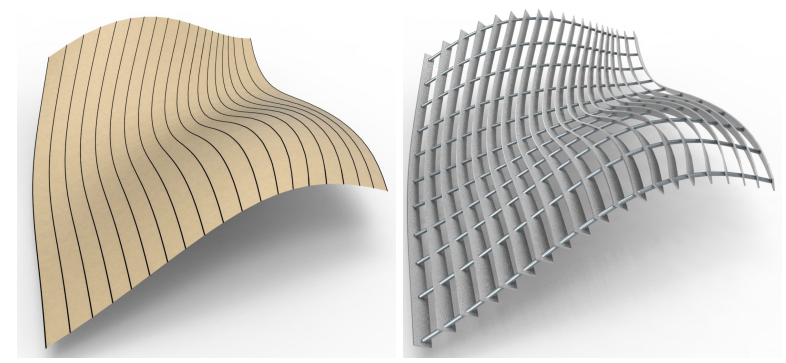

Fig. 6. Pseudo-geodesics. At left, we show a surface covered by almost equidistant pseudo-geodesics, whose computation is discussed in §3.2. At right, we show the rectifying developable of each. It has constant angle with the reference surface (by the pseudo-geodesic property) and unfolds to a straight strip (by the rectifying property). One can imagine this arrangement of strips as a shading system. 

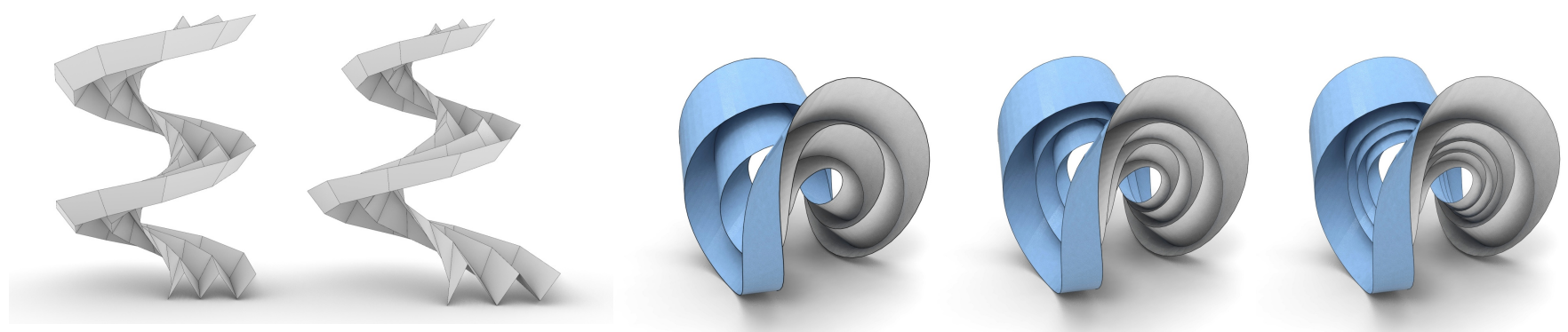

Fig. 7. Mesh parallelity for design of pleated structures. At left, we show two parallel discrete pleated structures, one of them with helical symmetry, the other one with spiral symmetry. This is an example where creases have constant slope w.r.t. a reference surface - a property not destroyed by mesh parallelism. At right, we show a sequence of pleated structures which undergoes subdivision based on mesh parallelism.

that corresponding edges $\mathbf{v}_{i} \mathbf{v}_{j}$ and $\overline{\mathbf{v}}_{i} \overline{\mathbf{v}}_{j}$ are parallel. Parallelity means that $\mathbf{v}_{i}-\mathbf{v}_{j}=\lambda_{i, j}\left(\overline{\mathbf{v}}_{i}-\overline{\mathbf{v}}_{j}\right)$. If in addition, $\lambda_{i, j}>0$, then the faces in $\bar{F}$ will automatically be proper planar quads, and the vertices of $\bar{V}$ will enjoy the developability condition (2). An appropriate limit shows an analogous statement for smooth pleated structures.

Design of discrete pleated structures. Mesh parallelism has several direct applications relevant to geometric design of PLS: We describe some procedures for their modification and construction. In each case we can invoke parallelism to see that the angle sum in vertices is $2 \pi$ throughout.

- $\quad$ Pleated structures from Cauchy initial values. For any given discrete PLS we may change two transverse mesh polylines arbitrarily as long as they remain

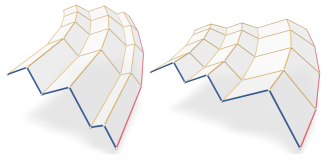
parallel to their respective originals (cf. blue and red polylines in the inset figure). This modification uniquely propagates to a quad mesh parallel to the first one. It will be discrete PLs if all factors $\lambda_{i, j}$ remain positive, see Fig. 7.

- Local refinement. We can replace a single fold by three consecutive folds in a completely local way using parallelism.

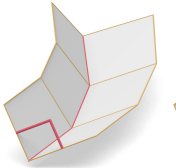

Locally around one vertex we modify a zigzag polyline by introducing a new zigzag (red in the inset) which we then propagate along a transverse polyline by parallel projection. An analogous construction for smooth PLS is shown by Tachi [2013]. Examples of parallel pleated structures are shown by Fig. 7 .

\subsection{Discrete principal pleated structures}

Recall that a smooth PLS is principal, if the fold angle along each crease is constant. Equivalent characterizations are existence of a right circular cone tangent to the developables to either side of the crease (Fig. 4), or the reflection-ruling property. A structurally consistent discretization of this property is the requirement that in each vertex there is a cone tangent to all four incident faces. In terms of the angles in a vertex, this is expressed as

$$
\omega_{1}+\omega_{3}=\omega_{2}+\omega_{4}
$$

[Liu et al. 2006]. If the developability condition (2) is assumed, this is further equivalent to

$$
\omega_{1}+\omega_{3}=\pi, \quad \omega_{2}+\omega_{4}=\pi .
$$

That condition is well known in origami and expresses flat-foldability of each vertex, i.e., besides the unfolding (all dihedral angles are zero) there is another flat configuration where some dihedral angles are $180^{\circ}$ [Huffman 1976]. According to Kokotsakis [1933], any such PLS has a continuous unfolding, during which the faces remain rigid and the edges act as hinges; see also [Tachi 2009].

Discrete PPLS are conical meshes, which are known to approximate the principal curvature lines of surfaces, provided the mesh polylines approximate the surface in a reasonable way. This is not the case for PPLS, since one half of the mesh polylines is zigzagging. We therefore cannot infer that mesh polylines approximate principal curvature lines. Nevertheless, we can draw on the full range of nice properties of conical meshes which are well established.

It is remarkable how well the geometric properties of a smooth PPLS are preserved by the discrete PPLS. We discuss several properties in the following paragraphs.

Construction of general developable conical vertices. Consider a vertex $v$ with four incident faces $f_{0}^{-}, f_{0}^{+}, f_{1}^{-}, f_{1}^{+}$and mountain and valley folds as shown by Fig. 8 . Here + and - refer to the two sides of a crease. The faces are contained in planes $T_{0}^{-}, T_{0}^{+}, T_{1}^{-}, T_{1}^{+}$, and the angles in these faces are denoted by $\omega_{1}, \ldots, \omega_{4}$ as shown by Fig. 8. By the conical property, all four planes are tangent to a certain cone associated with $v$. There are rotations about the axis of this cone which map $T_{0}^{-} \rightarrow T_{1}^{-}$and $T_{0}^{+} \rightarrow T_{1}^{+}$. If these rotations are equal, then it follows immediately that the vertex is developable, see Fig. 8. If they are different, i.e., if the 1-ring neighbourhood of the vertex $v_{1}$ is not constructed as in Fig. 8, then $\omega_{1}+\omega_{3} \neq \pi$, and the vertex is not developable.
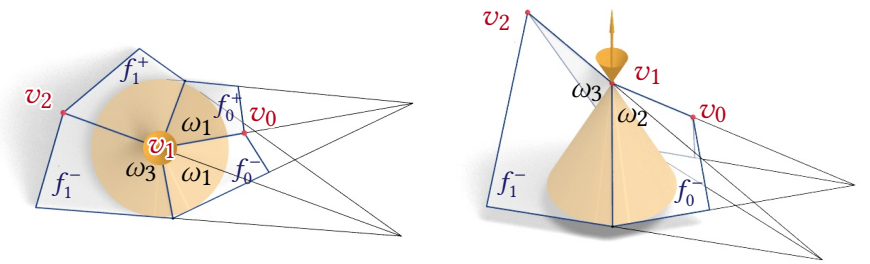

Fig. 8. Geometry of a developable conical vertex $v_{1}$ which is part of a crease $v_{0} v_{1} v_{2} \ldots$ (top view and side view). Faces $f_{0}^{-}, f_{0}^{+}$lie in two tangent planes of the cone associated with the vertex $v_{1}$, and rotation of these planes about the cone's axis yields planes carrying faces $f_{1}^{-}, f_{1}^{+}$. Observe that the angle $\omega_{1}$ occurs twice in the top view figure, proving $\omega_{1}+\omega_{3}=\pi$. 

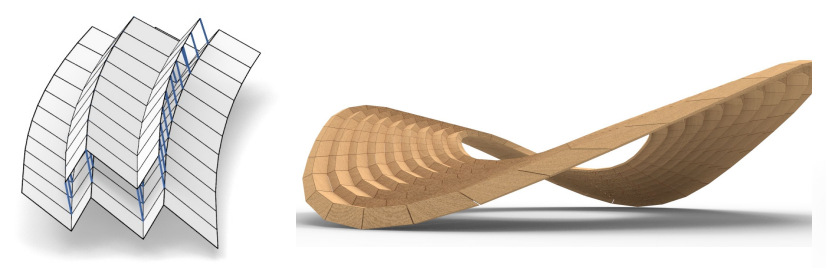

Fig. 9. Discrete principal pleated structures are conical meshes and have offsets at constant distance. Right: A wooden construction of constant thickness based on a PpLs. Observe the clean intersection of elements in the nodes which does not occur if the guiding mesh fails to be conical.

Constant fold angles along polylines. The previous paragraph shows that the angle between faces $f_{0}^{-}, f_{0}^{+}$equals the angle between $f_{1}^{-}, f_{1}^{+}$. By repeating the argument for successive vertices $v_{2}, v_{3}, \ldots$ along a crease, we conclude that the signed fold angle is constant along a crease.

The same argument shows that the dihedral angle between faces $f_{0}^{-}, f_{1}^{-}$is equal in magnitude to the dihedral angle between faces $f_{0}^{+}, f_{1}^{+}$, but has opposite sign. This fact is known in origami.

Rectifying developable. The cone axes of successive vertices $v_{i}$, $v_{i+1}$ are co-planar (this is true for any conical mesh, cf. [Liu et al. 2006]), and these axes constitute a discrete developable surface $R$. From the symmetry properties shown by Fig. 8 it follows that unfolding the auxiliary developable $R$ (not the PLs itself) maps the crease $v_{0} v_{1} v_{2} \ldots$ to a straight line. This makes $R$ a discrete version of the rectifying developable shown by Fig. 3. The same symmetries imply that faces $f_{i}^{-}, f_{i}^{+}$lie symmetric w.r.t. $R$, which is yet another analogy to the smooth case.

Offsets. Conical meshes are characterized as those meshes which have offsets at constant face-face distance. This leads to a nice characterization: discrete PPLS are characterized as those PLS which admit offsets at constant face-face distance. Fig. 9 shows how to exploit this property in practice.

The relation between a PPLS and its offset is a special case of mesh parallelity. The principal property is defined via angles and thus is not destroyed by mesh parallelism.

Like any conical mesh, a PPLS and its offsets have the same cone axes. Corresponding polylines are connected by discrete developables (the ones along the crease curves are the rectifying developables mentioned earlier).

\subsection{Flexion of principal-pleated structures}

So far, the discrete principal-pleated structures have proved a good discretization of the smooth case. There is one more important property which proves this, namely 1-DOF flexibility.

Existence of a continuous and unique (up to rigid motions) unfolding is known from previous results, e.g. [Kokotsakis 1933]. During the unfolding of a vertex star as shown by Fig. 10, angles between edges remain unchanged and conditions (2), (4) remain in force. Thus a PPLS remains principal during the unfolding.

It is known how any fold angle evolves during a continuous folding, cf. [Tachi 2010a]. In the notation of Fig. 8, with $\phi_{1}, \phi_{2}$ being the fold angles along edges $f_{0}^{-} \cap f_{0}^{+}$resp. $f_{0}^{-} \cap f_{1}^{-}$,we have

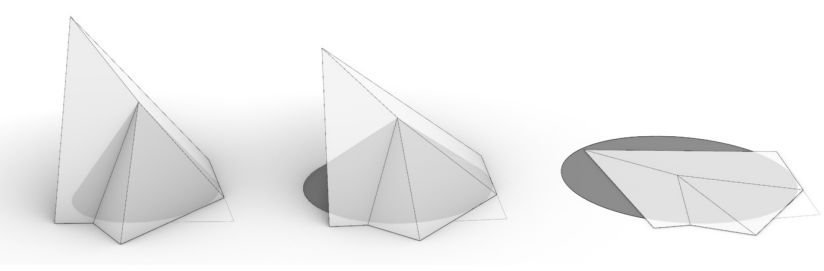

Fig. 10. Continuous unfolding of a single vertex in a principal-pleated structure. The vertex remains conical and developable throughout the process; the cone tangentially inscribed in the vertex star is not deforming isometrically: it opens up and becomes flat.

$\frac{\tan \phi_{2} / 2}{\tan \phi_{1} / 2}=\frac{\sin \left(\omega_{2}-\omega_{1}\right) / 2}{\sin \left(\omega_{2}+\omega_{1}\right) / 2}$, which is constant over time. By iteration, the analogous ratio involving any two fold angles in the PPLS is constant. Thus, for a continuous unfolding over time $t$, a fold angle $\phi(t)$ changes according to $\tan \frac{1}{2} \phi(t)=\lambda(t) \cdot \tan \frac{1}{2} \phi\left(t_{0}\right)$, where $t_{0}$ is some fixed reference time instant. The factor $\lambda(t)$ applies to all edges simultaneously. We emphasize this because it shows that the flexing motion of PPLS has exactly 1 degree of freedom.

\subsection{The relation of pleats to pseudo-geodesics}

Our major goal is the reconstruction of surfaces by means of pleated structures, in particular ones where the folds have uniform width. This can only be done by numerical optimization, which requires a suitable initialization.

Consider first a pleated structure with a small distance between creases which lie below and above a reference surface in an alternating way. The creases follow smooth guiding curves on the surface. An upright folding such as in Fig. 11, left, has crease curves whose rectifying developable is orthogonal to the reference surface, while the osculating planes are parallel to it. This property characterizes asymptotic curves. We conclude that upright folds follow asymptotic curves, which exist only on negatively curved surfaces. There is no design freedom except to choose between the two families of asymptotic curves, and their spacing.

The limit, as the width of folds approaches zero, has been computed explicitly by [Dias et al. 2012] for the case of the pleated annulus, see e.g. [Demaine et al. 2011b]. Limits of creases are indeed asymptotic curves, and Gaussian curvature $K$ and torsion $\tau$ of creases in the limit are related by $\tau=\sqrt{-K}$ (this is a known property of asymptotic curves).

In order to obtain more design freedom, and to be able to treat positively curved surfaces, we need another idea. We think of inclined folds as in Fig. 11, right. In this case the rectifying developable

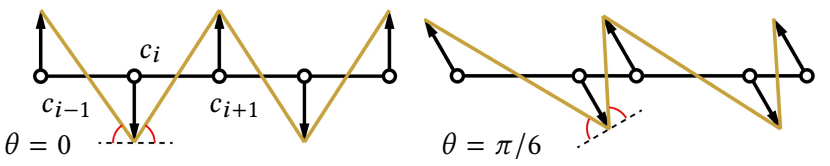

Fig. 11. Schematic illustration of a zigzag pleated structure following a reference surface. Creases follow curves $c_{i}$ on the reference surface. The rectifying developables of creases are indicated by arrows. They act as angle bisectors at creases, and their angle $\theta$ with the surface normals is used to guide the relative position of pleats and reference surface. 


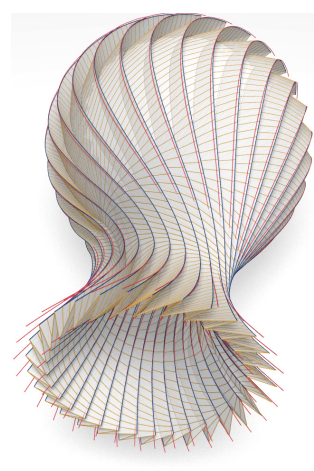

Fig. 12. If the inclination angle of a fold w.r.t. the reference surface does not change along the fold, then this fold must follow a pseudogeodesic curve. Therefore pseudo-geodesics can be used to initialize the optimization procedure we use to compute pleated structures. In this image the creases computed via optimization are shown in blue. Optimization has been initialized by the red curves which are created by moving pseudo-geodesics out of the surface. In the notation of Fig. 11, all folds have inclination angle $\theta=\pi / 3$.

of crease curves has a constant angle with the reference surface, making the creases pseudo-geodesics (see § 2.2).

We do not formalize this limit process, since we are using it only as an idea for initialization. An example, computed with the algorithm presented in $\S 3$, is shown by Fig. 12. In the extreme case of folds being actually tangent to the reference surface, the creases follow geodesics. This happens e.g. for Jun Mitani's folded sphere designs [Mitani 2009b].

The constant inclination requirement is arbitrary, even if we think it is sensible. It allows us to reduce the layout of pleats to the computationally manageable task of patterns of pseudo-geodesics. Dropping this requirement, we have greater design freedom. It is however not easy to exploit this freedom, since we no longer have such a nice class of guiding curves for the pleats. An arbitrary arrangement of guiding curves will not work, as demonstrated by Fig 25 .

\section{ALGORITHMS FOR DESIGN AND RECONSTRUCTION}

Having established a connection between pleated structures and pseudo-geodesics, we propose the following three-step procedure for their reconstruction and design. The first step is to create a pattern of pseudogeodesic guiding curves on a surface. For reconstruction, this is a given reference surface (§ 3.1). For design we create the surface simultaneously with the curve pattern (see §3.2).

The second step is to initialize optimization by moving the guiding curves away from the reference surface as shown in Fig. 11 to obtain initial guesses for creases. We lay out a quad mesh along these creases. See $\S 3.3$ for details.

Thirdly, we optimize this mesh for planarity of quads, developability, and also for the principal property if applicable - see $\S 3.4$.

\subsection{Tracing pseudo-geodesics on surfaces}

For creating patterns of guiding curves, tracing a pseudo-geodesic curve on a reference mesh $M=(V, E, F)$ is a basic task. This is an initial value problem analogous to the shooting of geodesic curves from an initial point in an initial direction. Fig. 13 explains how we do it. The curve is represented as a polyline with vertices $\mathrm{p}_{i}$. The pseudo-geodesic property says its osculating planes (spanned by consecutive vertices $\mathbf{p}_{i}, \mathbf{p}_{i+1}, \mathbf{p}_{i+2}$ ) enclose the angle $\theta$ with $M$. We also know if we are inclined to the left or the right, as we progress along the polyline. We start with an edge $\mathbf{p}_{i}, \mathbf{p}_{i+1}$ where $\mathbf{p}_{i}$ lies
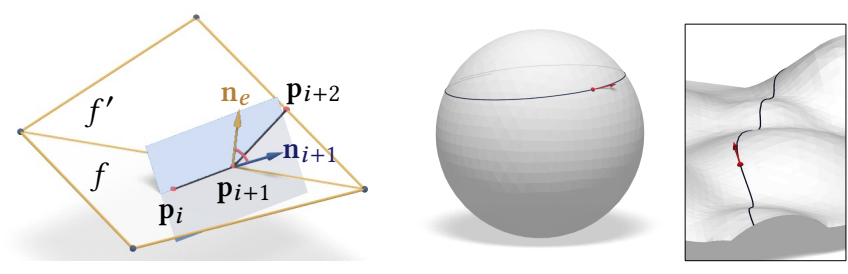

Fig. 13. Tracing pseudo-geodesics. We require that the plane spanned by vertices $\mathrm{p}_{i}, \mathrm{p}_{i+1}, \mathrm{p}_{i+2}$ is inclined by the angle $\theta$ w.r.t. the reference surface (where the normal vector $\mathbf{n}_{e}$ of the reference surface is an average of face normals computed in $\left.\mathrm{p}_{i+1}\right)$. Center and Right: Some test cases with $\theta=\pi / 3$. In the sphere, all pseudo-geodesics are closed.

in a face $f$ and $\mathbf{p}_{i+1}$ is contained in the edge $f \cap f^{\prime}$. Face normal vectors $\mathbf{n}_{f}, \mathbf{n}_{f^{\prime}}$ yield a surface normal vector $\mathbf{n}_{e}=\mathbf{n}_{f}+\mathbf{n}_{f^{\prime}}$ in $\mathbf{p}_{i+1}$. We now compute a normal vector $\mathbf{n}_{i+1}$ of the osculating plane $\mathbf{p}_{i} \mathbf{p}_{i+1} \mathbf{p}_{i+2}$ by requiring $\varangle\left(\mathbf{n}_{e}, \mathbf{n}_{i+1}\right)=\theta$. We select the right one of two solutions via the sign of $\operatorname{det}\left(\mathbf{n}_{e}, \mathbf{p}_{i+1}-\mathbf{p}_{i}, \mathbf{n}_{i+1}\right)$. Now $\mathbf{p}_{i+2}$ is uniquely determined as intersection of the osculating plane with the boundary of face $f^{\prime}$. From here the procedure continues recursively.

The design of a pattern is illustrated by Figure 2: We cross the surface with a suitable curve (e.g., a geodesic), and from regularly spaced points on $c$ shoot pseudo-geodesics orthogonal to $c$. Similar to the case of geodesics [Pottmann et al. 2010], we cannot expect anything like exactly equidistant pseudo-geodesics.

\subsection{Generating surfaces by evolution of pseudo-geodesics}

The goal here is to generate a sequence of nearly equidistant pseudogeodesics on a surface, which emerges during this process. We describe how to construct a polygon $\left\{\mathbf{v}_{n+1, i}\right\}_{i \in \mathbb{Z}}$ from its predecessor $\left\{\mathbf{v}_{n, i}\right\}_{i \in \mathbb{Z}}$. By applying this procedure iteratively, we generate a discrete surface from any initial polygon $\left\{\mathbf{v}_{0, i}\right\}_{i \in \mathbb{Z}}$.

We endow the vertex $\mathbf{v}_{n, i}$ with a discrete Frenet frame $\mathrm{e}_{n, i}^{k}$, $k=1,2,3$, see Fig. 14 . We choose the binormal vector $\mathbf{e}_{n, i}^{3}$ orthogonal to the osculating plane spanned by three consecutive vertices $\mathbf{v}_{n, i-1} \mathbf{v}_{n, i} \mathbf{v}_{n, i+1}$ and letting the unit tangent vector $\mathbf{e}_{n, i}^{1}$ equal the normalized vector $\mathbf{v}_{n, i+1}-\mathbf{v}_{n, i-1}$.

To compute $\mathbf{e}_{n, i}^{3}$ in a stable way, we perform optimization. We minimize $\sum_{i}\left\langle\mathbf{v}_{n, i+1}-\mathbf{v}_{n, i}, \mathbf{e}_{n, i}^{3}\right\rangle^{2}+\left\langle\mathbf{v}_{n, i-1}-\mathbf{v}_{n, i}, \mathbf{e}_{n, i}^{3}\right\rangle^{2}+\left(\left\|\mathbf{e}_{n, i}^{3}\right\|^{2}-\right.$ $1)^{2}$, together with a small dose of the regularizer $\sum_{i} \| \mathbf{e}_{n, i+1}^{3}+$

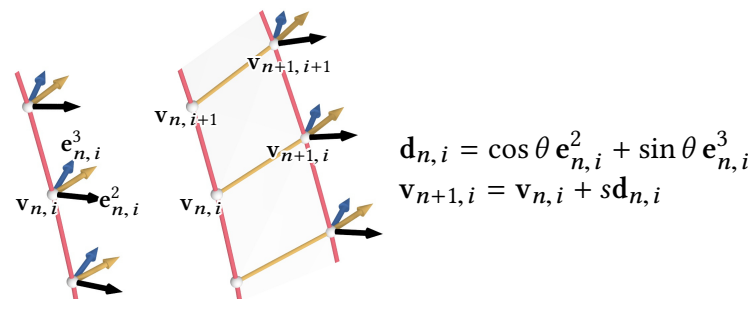

Fig. 14. Evolution of pseudo-geodesics. If a polyline $\left\{\mathbf{v}_{n, i}\right\}_{i \in \mathbb{Z}}$ evolves by the formulae shown above, it is a discrete pseudo-geodesic in the surface generated by the evolution process. Here $\mathbf{e}_{n, i}^{1}, \mathbf{e}_{n, i}^{2}, \mathbf{e}_{n, i}^{3}$ is the discrete Frenet frame associated with the vertex $\mathrm{v}_{n, i}$, and $s$ is a distance. 

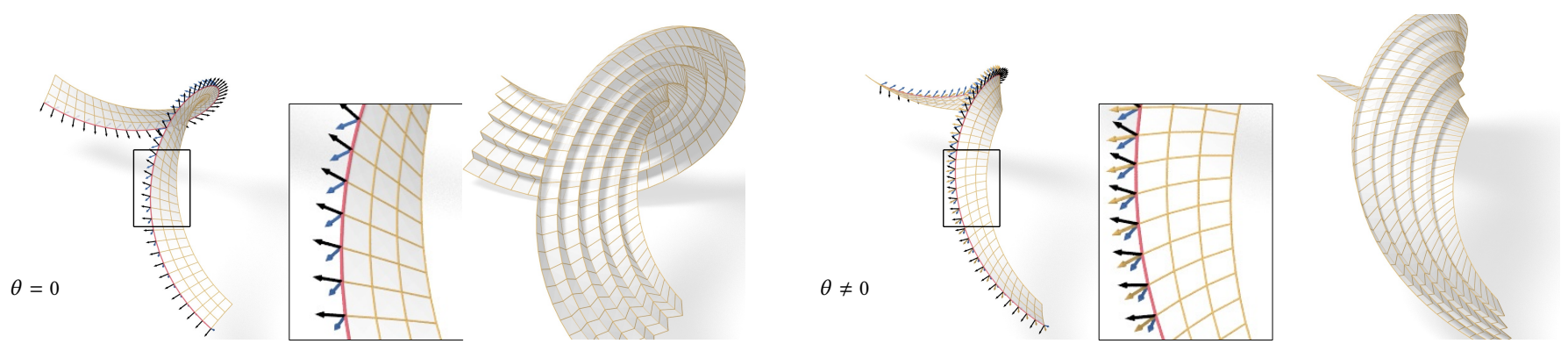

Fig. 15. Two examples of pleated structures generated from pseudo-geodesics evolving with constant speed. The left hand example has $\theta=0$ and corresponds to evolution along principal normals $\mathbf{e}_{j, i}^{2}$. This generates a surface with an equidistant family of asymptotic curves and, in turn, a pleated structure with upright folds. The example at right has inclined folds.

$\mathbf{e}_{n, i-1}^{3}-2 \mathbf{e}_{n, i}^{3} \|^{2}$. We solve this small optimization problem in the same way as our main optimization discussed in $\S 3.4$.

This evolution equation which computes the next row of vertices $\mathbf{v}_{n+1, i}$ is shown by Fig. 14. Consider now the discrete surface with vertices $\mathbf{v}_{n, i}$ and the canonical quad mesh combinatorics. By construction, the row and column mesh polylines discretize curves intersecting orthogonally, such that osculating planes of the first family has angle $\theta$ with the surface. The polylines $\left\{\mathbf{v}_{n, i}\right\}_{i \in \mathbb{Z}}$ therefore represent a family of pseudo-geodesics.

Fig. 15 shows some examples. The angle $\theta$ may depend on $n$, and the distance $s$ can even depend on both $i$ and $n$ - see Figs. 16, 27.

\subsection{Initializing a discrete surface with creases}

Having created a pattern $\mathbf{v}_{l, i}$ of discrete pseudo-geodesics according to $\S 3.2$, we connect these vertices to form a quad mesh $M_{0}=$ $\left(V_{0}, E, F\right)$ in the obvious way. We then generate a zigzag offset surface as shown by Fig. 11, by moving each pseudo-geodesic off the surface in an alternating way. The distance of crease curves from the reference surface influences the positioning of rulings - a small distance implies that rulings are almost tangential to the creases and singularities might develop.

Figure 11 reveals that uniform spacing of creases does not correspond to uniform spacing of pseudo-geodesics on the reference surface. It would not be difficult to account for this in our initialization, but actually this is not necessary. Subsequent optimization works just as well if we move uniformly spaced pseudo-geodesics in direction of the binormal vectors $\mathrm{e}_{n, i}^{3}$ to initialize creases.

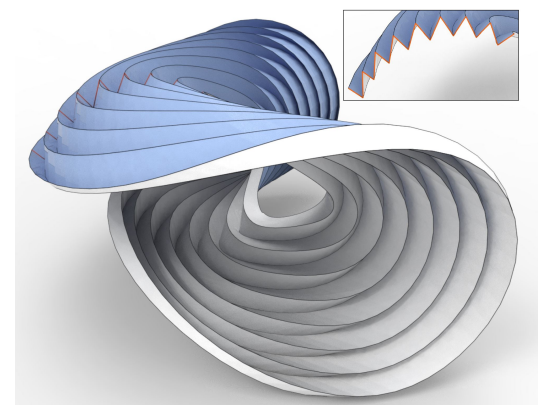

Fig. 16. Variations of pseudo-geodesic patterns. This optimized pleated structure has been initialized from pseudogeodesics whose inclination angle $\theta$ is varying from one curve to the next.
The presentation so far only deals with a very simple arrangement of folds, namely a sequential one. We show more complicated examples with combinatorial singularities below. In surfaces generated by evolution, we used the simplest of all possible combinatorics and did not experiment with elementary modifications like cropping.

We end up with a quadrilateral mesh $M_{1}=\left(V_{1}, E, F\right)$ whose vertices are already positioned above and below a reference surface, with crease curves following pseudo-geodesics. The faces of the mesh are far from being planar, which is a property to be established via optimization. So is developability.

As variables for optimization we use vertices $\mathbf{v}_{i}, \overline{\mathbf{v}}_{i}$ of the mesh and its unfolding, as well as normal vectors $\mathbf{n}_{f}$ of faces $f \in F$.

\subsection{Optimization}

The variables introduced in $\$ 3.3$ obey a number of constraints. Firstly, for each face we penalize deviation from planarity: Letting

$$
E_{\text {plan }}=\sum_{f \in F} \sum_{\mathbf{v}_{i} \mathbf{v}_{j} \subset f}\left\langle\mathbf{v}_{i}-\mathbf{v}_{j}, \mathbf{n}_{f}\right\rangle^{2}+\sum_{f \in F}\left(\left\|\mathbf{n}_{f}\right\|^{2}-1\right)^{2},
$$

smallness of $E_{\text {plan }}$ means that each normal vector has length 1, and any edge $\mathbf{v}_{i} \mathbf{v}_{j}$ of a face $f$ is orthogonal to $\mathbf{n}_{f}$.

Secondly, developability of a mesh $M$ is expressed by existence of another mesh $\bar{M}$ (the unfolding of $M$ ) which is contained in a plane, and which is both combinatorially equivalent and isometric to $M$. Every vertex $\mathbf{v}_{i}$ has a corresponding vertex $\overline{\mathbf{v}}_{i}$ in the unfolding, where $\overline{\mathbf{v}}_{i} \in \mathbb{R}^{2}$. The mesh and its unfolding are isometric, if the energy $E_{\text {isom }}$ vanishes, where

$$
E_{\text {isom }}=\sum_{\substack{\text { edges and diagonals } \\ \mathbf{v}_{i} \mathbf{v}_{j} \text { of faces }}}\left(\left\|\mathbf{v}_{i}-\mathbf{v}_{j}\right\|^{2}-\left\|\overline{\mathbf{v}}_{i}-\overline{\mathbf{v}}_{j}\right\|^{2}\right)^{2} .
$$

Creases in the mesh are represented by polylines. We require that in the course of optimization, vertices move mostly along those polylines. To ensure that, we compute for each vertex $\mathbf{v}_{i}$ the closest point projection onto its polyline, and we also determine the principal normal vector $\mathbf{n}_{i}^{*}$ there (as an average of the principal normal vectors $\mathbf{e}_{n, i}^{2}$ we computed before). The energy

$$
E_{\text {close }}=\sum_{\mathbf{v}_{i} \in V}\left\langle\mathbf{v}_{i}-\mathbf{v}_{i}^{*}, \mathbf{n}_{i}^{*}\right\rangle^{2}
$$

penalizes movement of vertices across creases, but does not penalize movement of vertices along the crease, or a change in the distance 


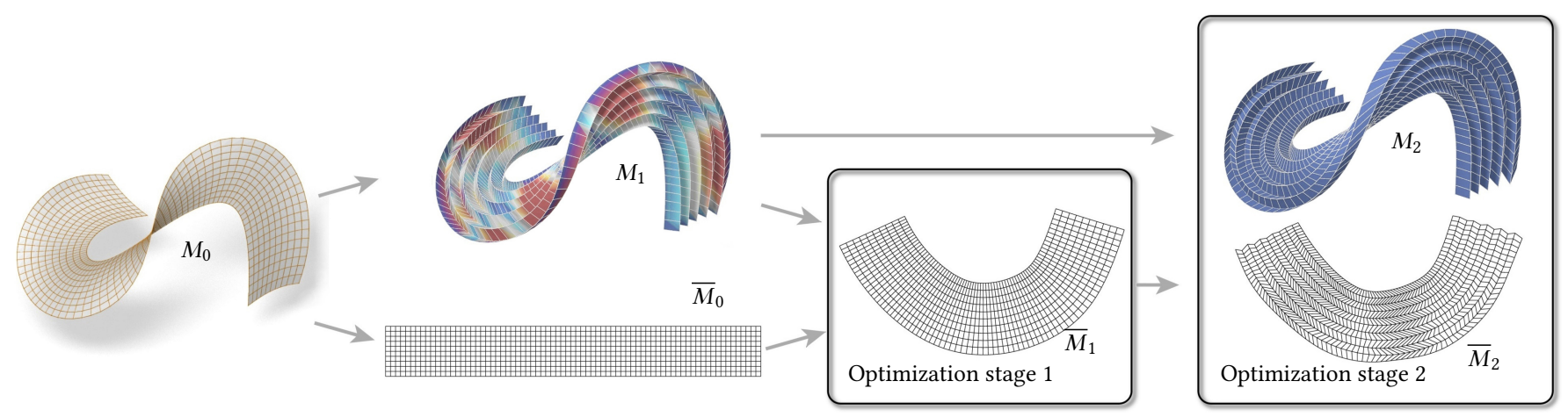

Fig. 17. Computation pipeline. A discrete pleated structure $M_{2}$ and its unfolding $\bar{M}_{2}$ are computed by a combined optimization procedure. Propagation of pseudo-geodesic curves produces a reference mesh $M_{0}$. Moving vertices off the reference surface yields a mesh $M_{2}$ exhibiting the correct creases already. Optimization stage 1 computes a quasi-unfolding $\bar{M}_{1}$ of $M_{1}$. Optimization Stage 2 achieves planarity of faces and exact developability by optimizing the pleated structure $M$ and its development $\bar{M}$ simultaneously. The color coding shows the angular defect in vertices.

of the crease curves from the reference surface. The projection and normal vectors are recomputed after each round of iteration.

Finally, fairness is quantified by means of

$$
E_{\text {fair }}=\sum_{\substack{\text { successive } \\ \text { vertices } \mathbf{v}_{i-1} \mathbf{v}_{i} \mathbf{v}_{i+1}}}\left\|\mathbf{v}_{i+1}+\mathbf{v}_{i-1}-2 \mathbf{v}_{i}\right\|^{2},
$$

where the summation is over all triples of successive vertices $\mathbf{v}_{i-1}$, $\mathbf{v}_{i}, \mathbf{v}_{i+1}$ of creases. We found that this fairness energy also prevents foldovers. Note that none of these energies accounts for the resolution of the discretization - for optimization the relative magnitude of energies is relevant.

The optimization itself happens in two stages, see Fig. 17 for an overview. Initialization (§3.3) provides a mesh $M_{1}$ which already representes a creased surface. It has vertices $\mathbf{v}_{i}$. Before optimizing it further, in a first stage of optimization we provide an initial guess at the unfolding, resp. development $\bar{M}_{1}$ of $M_{1}$. For that, we start with a simple mesh $\bar{M}_{0}$, with vertices $\overline{\mathbf{v}}_{i}$, which is part of a square grid and combinatorially equivalent to $M_{0}$. Its edgelength is the average edgelength of $M_{1}$. We now minimize $E_{\text {isom }}$ with variables $\overline{\mathbf{v}}_{i}$ to create $\bar{M}_{1}$. The mesh $\bar{M}_{1}$ is, under the circumstances, as isometric as possible to $M_{1}$.

In the second stage of optimization, we achieve planarity of faces and developability by optimizing for $E_{\text {plan }} \rightarrow 0, E_{\text {isom }} \rightarrow 0$. To be

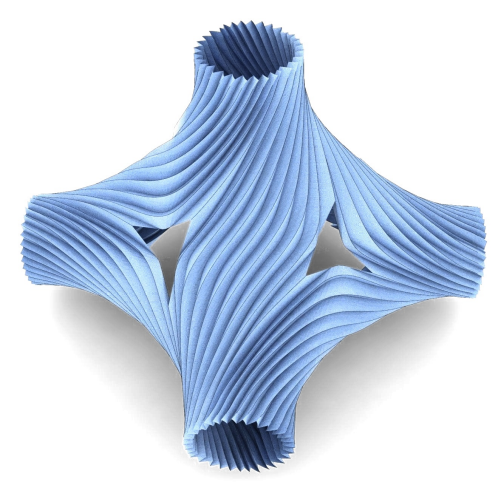

Fig. 18. Patterns with singularities. Among all pseudogeodesics, those with $\theta=$ 0 (asymptotic curves) offer the least design freedom, but also the possibility of patterns with singularities in the umbilics of the reference surface. The index of the asymptotic cross field is a multiple of $\frac{1}{2}$, enabling an alternating mountain/valley folding. precise, we use the objective function

$$
E=\lambda_{1} E_{\text {plan }}+\lambda_{2} E_{\text {close }}+\lambda_{3} E_{\text {isom }}+\lambda_{4} E_{\text {fair }}
$$

with weights chosen such that $E_{i s o m}, E_{\text {plan }}$ dominate. The choice of weights for the examples in this paper is shown in Fig. 21.

The principal property. If the pleated structure subject to optimization is to be principal, we have to ensure that the fold angle along a crease is constant. We consider all tuples $\left(f_{i}^{+}\right.$, $f_{i}^{-}, f_{i+1}^{+}, f_{i+1}^{-}$) of faces such that $f_{i}^{+} \cap f_{i}^{-}$is part

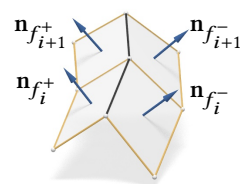
of a discrete crease, and $f_{i+1}^{+} \cap f_{i+1}^{-}$is the next edge on that crease, cf. Fig. 8. By summation over all these tuples we construct

$$
E_{\text {principal }}=\sum_{\left(f_{i}^{+}, f_{i}^{-}, f_{i+1}^{+}, f_{i+1}^{-}\right)}\left(\left\langle\mathbf{n}_{f_{i}^{+}}, \mathbf{n}_{f_{i}^{-}}\right\rangle-\left\langle\mathbf{n}_{f_{i+1}^{+}}, \mathbf{n}_{f_{i+1}^{-}}\right\rangle\right)^{2},
$$

and we add $\lambda_{5} E_{\text {principal }}$ to the target functional (5) whenever necessary. Examples are shown by Figures 1, 9, 26, and 27.

\section{RESULTS AND DISCUSSION}

The methods developed in this paper allow us to create surfaces which admit a nice pattern of curved folds, by free evolution of pseudo-geodesics, and derive pleated structures from them, see Figures 1, 9, 12, 15, 16, 19, 24, 27, 26. The characteristic angle of the pseudo-geodesics can be chosen between $0^{\circ}$ (asymptotic lines case) and $90^{\circ}$ (geodesic case).

Principal PPLS and mechanisms. In particular, we point to the special case of principal pleated structures, which enjoy continuous ruling-preserving unfolding, a flat-folded state, and constant fold angle along creases. It is this case where the analogy between the smooth and discrete cases is most gratifying. Examples are shown by Figures 1, 9, 26, and 27. Fig. 26 highlights the interesting fact that PPLS are mechanisms. If the number of edges and faces allows this, they can be built with rigid faces connected by hinges. The fact that our method has many degrees of freedom means that we have contributed to the design of mechanisms. 

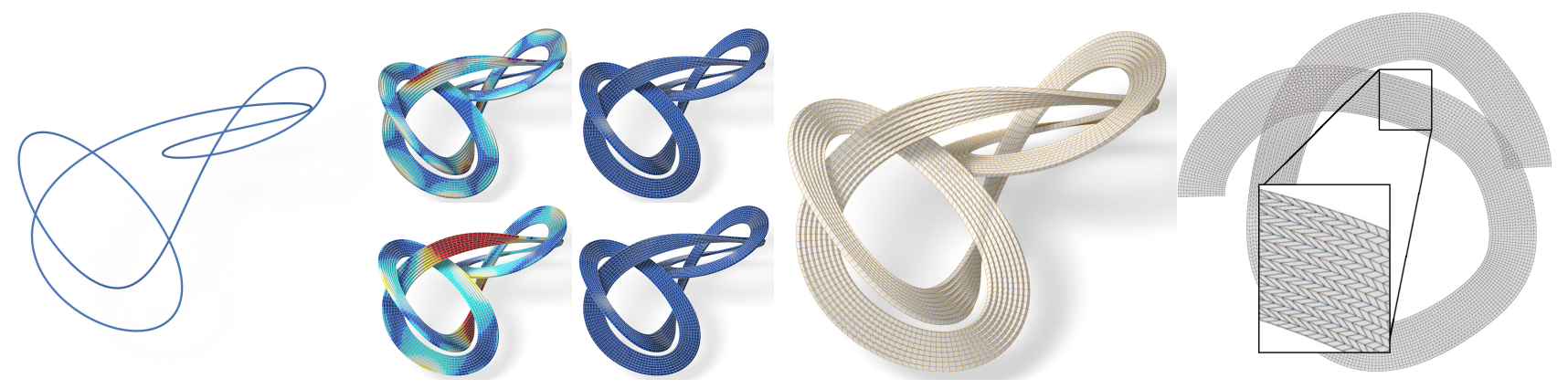

Fig. 19. Design example. From left: input curve; the process of initialization via evolution of pseudo-geodesics and optimization; the result of optimization; the development. The color-coded images show the planarity defect (top row) and angle defect (bottom row) before and after optimization. The unfolding demonstrates the fact that pleated structures need not be unfoldable in a 1-1 manner.

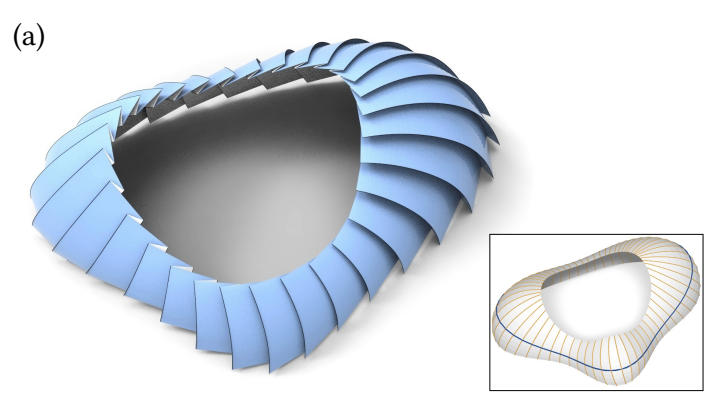

(b)

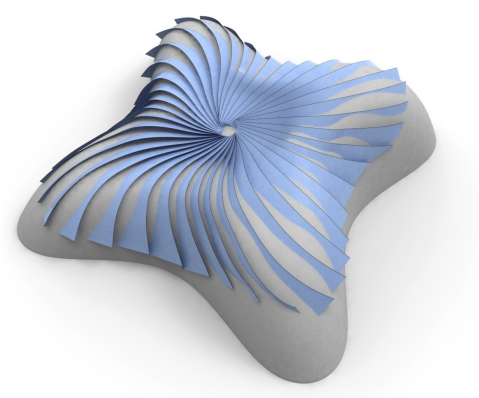

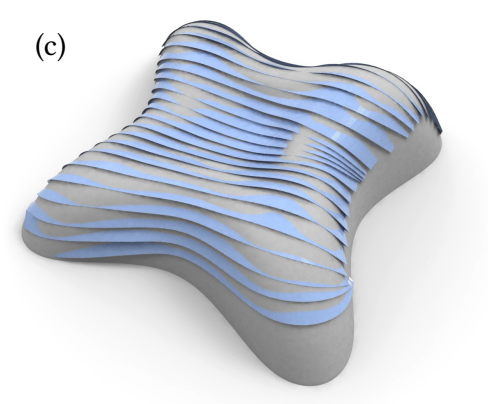

Fig. 20. Surface reconstruction examples. Diverse patterns can be created by shooting pseudo-geodesics orthogonal to a guiding curve (subfigures a,c) or simply by shooting pseudogeodesics from a chosen vertex point (subfigure b). This pattern of guiding curves then serves as the basis of a pleated structure approximating a given surface. The PLS in (b) and (c) use as reference surface the top part of the roof of the proposed Lilium tower by Zaha Hadid.

Fig. $|V| \quad|F| \lambda_{1} \lambda_{2} \lambda_{4} \lambda_{5} \quad w_{\text {planar }}(\max / \mathrm{av}) \quad w_{\text {angle }}(\max / \mathrm{av}) \quad \#$ it $\quad T$

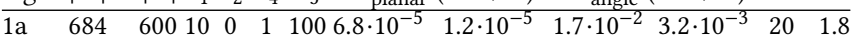

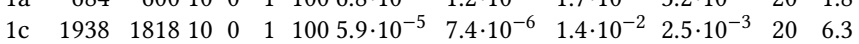

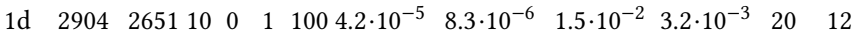

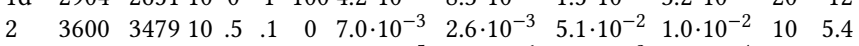

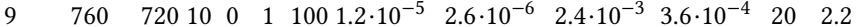

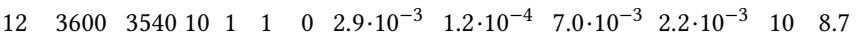

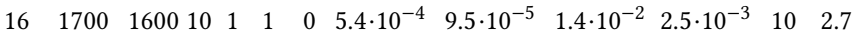

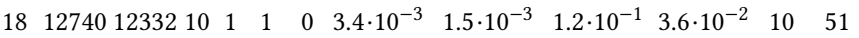

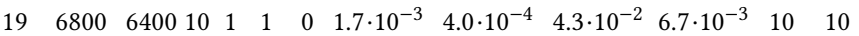

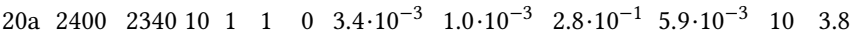
20b $4800 \begin{array}{lllllllllll}4720 & 1 & 1 & 0.5 & 0 & 1.4 \cdot 10^{-2} & 4.0 \cdot 10^{-3} & 1.4 \cdot 10^{-2} & 5.1 \cdot 10^{-3} & 10 & 10.1\end{array}$

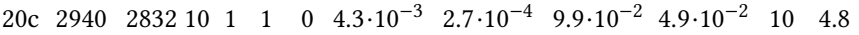
$\begin{array}{lllllllllllll}22 & 3027 & 288020 & 1 & 1 & 0 & 3.2 \cdot 10^{-3} & 3.0 \cdot 10^{-4} & 7.2 \cdot 10^{-2} & 8.1 \cdot 10^{-3} & 20 & 8.5\end{array}$

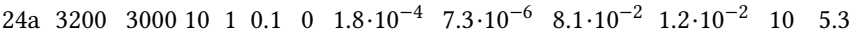

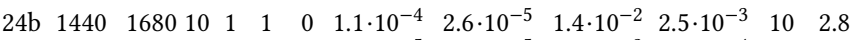

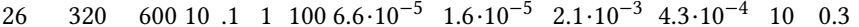

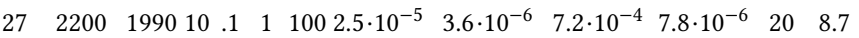

Fig. 21. Statistics. For the examples of this paper, we show the number of vertices and faces of the meshes involved, from which we deduce that our optimization involves $5|V|+3|F|$ variables. We also show the weights $\lambda_{1}, \ldots, \lambda_{5}$ which determine the individual contributions to the target functional (5) $\left(\lambda_{3}=100\right.$ always). The quality of meshes is measured by a planarity measure $w_{\text {planar }}$, which is the distance of diagonals in a quad divided by average length of those diagonals. The quality of developability is measured by the angle defect $w_{\text {angle }}$, which gives the discrepancy betwee the angle sum in a vertex and 360 degrees. We also show the number of iterations and the time needed (in seconds).
Reconstruction and Editing. As to approximation of reference surfaces, this is possible whenever we manage to lay out a pattern of pseudo-geodesics on them, see Figures 2 and 20. This is not so easy in presence of many smaller features, but the examples of Figures 2 and $20(b, c)$ show well the capabilities of the method.

If we do not care about controlling the inclination angle of folds, we have a lot more design freedom. Figure 23 shows what happens if we gently edit a reference shape and guiding curves lose the pseuogeodesic property: A pleated structure derived from them will have variable inclination but is fine otherwise. Optimization does fail, however, if the initialization does not take the folds' osculating planes into account, as demonstrated by Fig 25.

As to patterns with combinatorial singularities, recall that curved creases are guided by pseudo-geodesic curves, and complex crease patterns require analogous patterns of pseudo-geodesics. Finding those is not an easy task, which we can deduce from the known case of geodesics. Only in the case of upright folds (guided by asymptotic curves), the loss of design freedom offers a tractable approach: The uniqueness of asymptotic curves automatically leads to patterns with combinatorial singularities around umbilical points of the reference surface. The asymptotic curves have singularities of the same index as the principal curvature lines, namely integer multiples of $\frac{1}{2}$. Thus they cleanly separate into two families. Pleated structures based on this construction are seen in Figures 18 and 22. 

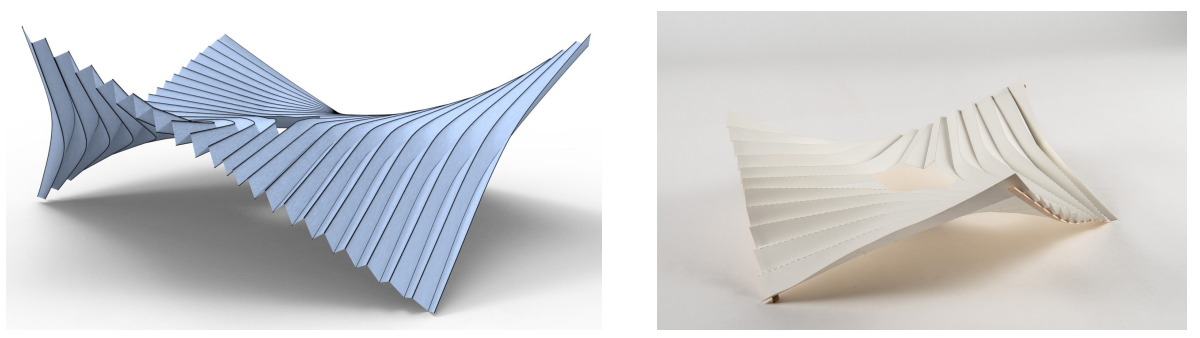

Fig. 22. By following the asymptotic curves on a negatively curved surface, we create a pleated structure with upright folding and combinatorial singularities. We show both a rendering and a photo of a paper model.

Relevance for fabrication. Fig. 9 illustrates the fact that principl PLS have offsets at constant face-face distance, which is highly relevant for constructions made from materials with nonzero thickness, like wood. Fig. 6 shows a structure made from curved elements whose unfolded state is straight, and which lie at constant angle to a reference surface. This application is interesting in the light of related research on curved elements from straight panels, cf. [Schling et al 2018].

Implementation Details. The target functional (5) is optimized by a Levenberg-Marquardt method. The implementation has been done in C++ using the data structures of OpenMesh, cf. [Botsch et al. 2002] and the TAUCs library for sparse linear solvers [Toledo 2003].

Detailed statistics are provided by the table in Fig. 21. These computation times refer to an Intel Xeon E5-2687W 3.0GHz processor without parallel processing or other acceleration techniques.

Limitations. The most important limitation of our method is that if we want to control the inclination angle of folds against the reference surface, it relies on patterns of pseudo-geodesic curves, and such patterns tend to fall into disarray when the reference surface has too many features. This is already the case for patterns of geodesics, whose distance is governed by the Jacobi equation [Pottmann et al. 2010]. Finding patterns of pseudo-geodesics is therefore a problem of its own. On more complicated surfaces we confined ourselves to upright foldings which follow asymptotic curves and are therefore available only in case of negative curvature. Another limitation is that we did not take the physics of folding paper into account - we only verified our computations a posteriori by building models.

Future research. There are several obvious avenues of future research. One possibility is to improve control resp. initialization in cases where the folds do not have nearly constant width. Another direction is the connection to discrete differential geometry: Discrete pleated structures are a non-smooth approximation of smooth surfaces, which is a rather unexplored area. We would even go so far as to call for new theories of discrete differential geometry which are entirely based on non-smooth approximations. Even existing origami constructions (e.g. Miura-Ori) could turn out to be special cases of a more general theory.

In this paper, the flexibility of pleats was exploited to solve a general approximation problem. We did not deal with the much more difficult problem of representing general shapes as curvedfolding surfaces with as few folds as possible.

Further, there are surely more connections to flat-foldable structures to be discovered, given e.g. the recent breakthrough in the classification of flexible quad meshes by [Izmestiev 2017]. Generally flexing geometry is a topic with high potential for interesting applications. It would be extremely interesting to realize flexible curve-pleated structures in architecture or building construction.

Conclusion. We have presented an analysis of curved folds, in particular curved folds of constant fold angle (this is the principal case), and we proposed discretizations exhibiting sufficient analogies to the smooth case to make their use for computation a reasonable choice. The discrete principal case has very interesting geometry. Establishing a relation between crease patterns on one hand, and patterns of pseudo-geodesic curves on the other hand, we show a method to create pleated structures of controlled fold width and inclination angle either by evolution, or following given design surfaces. Our method is, within limits, also capable of handling combinatorial singularities. Several properties, namely continuous foldability, and existence of offsets, are relevant for fabrication and applications, and we contribute to the design of flexible structures.

\section{ACKNOWLEDGMENTS}

This work was supported by the SFB-Transregio programme Discretization in geometry and dynamics, through grant I2978 of the Austrian Science Fund. Caigui Jiang and Florian Rist were supported by KAUST baseline funding.

\section{REFERENCES}

Thomas Barois, Loïc Tadrist, Catherine Quilliet, and Yoël Forterre. 2014. How a Curved Elastic Strip Opens. Phys. Rev. Letters 113 (2014), \#214301, 1-5.

M. Ben Amar and Y. Pomeau. 1997. Crumpled paper. Proc. R. Soc. London Ser A. 453 (1997), 729-755.

Alexander Bobenko and Yuri Suris. 2009. Discrete differential geometry: Integrable Structure. American Math. Soc.

Mario Botsch, Stephan Steinberg, Stephan Bischoff, and Leif Kobbelt. 2002. OpenMesh: A Generic and Efficient Polygon Mesh Data Structure. Proc. OpenSG Symposium.

Sebastien J.P. Callens and Amir A. Zadpoor. 2018. From flat sheets to curved geometries: Origami and kirigami approaches. Materials Today 21, 3 (2018), 241-264.

Enrique Cerda, Sahraoui Chaieb, Francisco Melo, and L. Mahadevan. 1999. Conical dislocations in crumpling. Nature 401 (1999), 46-49.

Enrique Cerda and L. Mahadevan. 1998. Conical surfaces and crescent singularities in crumpled sheets. Phys. Rev. Lett. 80 (1998), 2358-2361.

Yan Chen, Rui Peng, and Zhong You. 2015. Origami of thick panels. Science 349, 6246 (2015), 396-400.

Eli Davis, Erik D. Demaine, Martin L. Demaine, and Jennifer Ramseyer. 2013. Reconstructing David Huffman's Origami Tessellations. f. Mechanical Design 135 (2013), \#111010, 1-7.

Erik Demaine, Martin Demaine, Vi Hart, Gregory Price, and Tomohiro Tachi. 2011b. (Non)existence of pleated folds: how paper folds between creases. Graphs and Combinatorics 27 (2011), 377-397.

Erik Demaine, Martin Demaine, and Duks Koschitz. 2011a. Reconstructing David Huffman's Legacy in Curved-Crease Folding. In Origami ${ }^{5}$. A. K. Peters, 39-52.

Erik Demaine, Martin Demaine, Duks Koschitz, and Tomohiro Tachi. 2015b. A Review on curved Creases in Art, Design and Mathematics. Symmetry: Culture and Science 26 (2015), 145-161. 


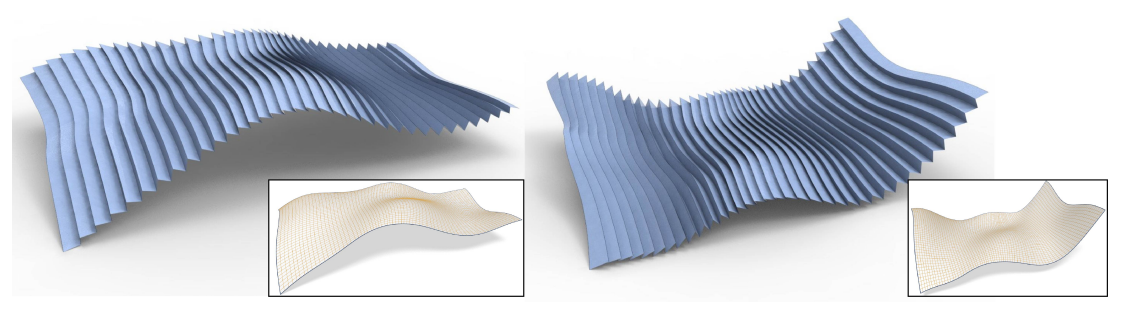

Fig. 23. Editing. Modification of the 'flying carpet' surface of Fig. 2 means the curves used for guiding creases are no longer pseudo-geodesic. Nevertheless the initialization of $\S 3.3$ yields folds capable of optimization. They no longer have constant inclination w.r.t. the reference surface. The success of this optimization depends on the fact that the guiding curves are not far from being pseudo-geodesic.
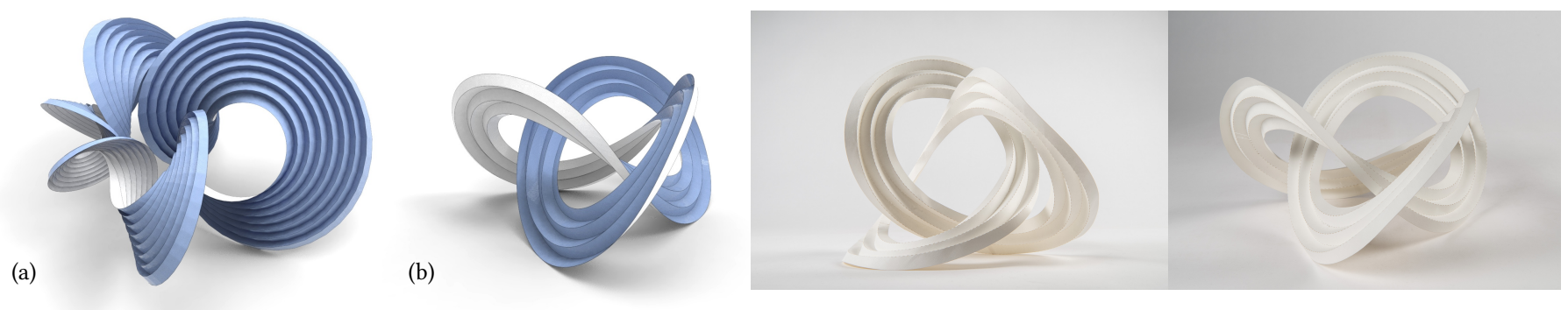

Fig. 24. Our paper is inspired by curved-folding art, and these examples in particular have been motivated by Erik and Martin Demaine's curved-folding sculptures. The pleated structures shown here have been computed by propagating pseudo-geodesics from a space curve in the manner of Fig. 19. The paper model has been made on the basis of the development we computed as part of our optimization procedure.

Erik D. Demaine, Martin L. Demaine, David A. Huffman, Duks Koschitz, and Tomohiro Tachi. 2015a. Characterization of Curved Creases and Rulings: Design and Analysis of Lens Tessellations. In Origami 6. Vol. 1. American Math. Soc, 209-230.

Erik D. Demaine, Martin L. Demaine, David A. Huffman, Duks Koschitz, and Tomohiro Tachi. 2018. Conic Crease Patterns with Reflecting Rule Lines. In Origami 7. Vol. 2. Tarquin, 574-590.

Erik D. Demaine, Martin L. Demaine, and Joseph S.B. Mitchell. 2000. Folding flat silhouettes and wrapping polyhedral packages: New results in computational origami Comp. Geom. 16 (2000), 3-21.

Erik D. Demaine and Joseph O'Rourke. 2007. Geometric Folding Algorithms. Cambridge University Press.

Erik D. Demaine and Tomohiro Tachi. 2017. Origamizer: A Practical Algorithm for Folding Any Polyhedron. In Proc. 33d Int. Symp. Comput. Geometry. \#34, 1-15.

Marcelo Dias, Levi Dudte, L. Mahadevan, and Christian Santangelo. 2012. Geometric Mechanics of Curved Crease Origami. Phys. Rev. Lett. 109, 114301 (2012), 1-13.

Marcelo A. Dias and Basile Audoly. 2014. A non-linear rod model for folded elastic strips. 7. Mechanics and Physics of Solids 62 (2014), 57-80.

Marcelo A Dias and Basile Audoly. 2015. Wunderlich, meet Kirchhoff: A general and unified description of elastic ribbons and thin rods. F. Elasticity 119 (2015), 49-66.

Marcelo A. Dias and Christian D. Santangelo. 2012. The shape and mechanics of curved-fold origami structures. Europhys. Lett. 100, 5 (2012), 54005.

Manfredo do Carmo. 1976. Differential Geometry of Curves and Surfaces. Prentice-Hall.

Yuntong Du, Changping Song, Jian Xiong, and Linzhi Wu. 2019. Fabrication and mechanical behaviors of carbon fiber reinforced composite foldcore based on curvedcrease origami. Composites Sc. \& Technology 174 (2019), 94-105.

Levi H. Dudte, Etienne Vouga, Tomohiro Tachi, and L. Mahadevan. 2016. Programming curvature using origami tessellations. Nature Materials 15 (2016), 583-588.

Thomas A. Evans, Robert J. Lang, Spencer P. Magleby, and Larry L. Howell. 2015a. Ridigly foldable Origami Twists. In Origami ${ }^{6}$. Vol. 1. American Math. Soc, 119-130.

Thomas A. Evans, Robert J. Lang, Spencer P. Magleby, and Larry L. Howell. 2015b. Rigidly foldable origami gadgets and tessellations. Royal Society Open Science 2 (2015), \#150067, 2-18.
William Frey. 2004. Modeling buckled developable surfaces by triangulation. ComputerAided Des. 36, 4 (2004), 299-313.

Dmitry Fuchs and Serge Tabachnikov. 1999. More on Paperfolding. Americal Math. Monthly 106 (1999), 27-35.

Matthew Gardiner, Roland Aigner, Hideaki Ogawa, and Rachel Hanlon. 2018. Fold Mapping: Parametric Design of Origami Surfaces with Periodic Tessellations. In Origami 7 . Vol. 1. Tarquin, 105-118.

Joseph Gattas and Zhong You. 2015. The behaviour of curved-crease foldcores under low-velocity impact loads. Int. F. Solids and Structures 53 (2015), 80-91.

Amanda Ghassaei, Erik Demaine, and Neil Gershenfeld. 2018. Fast, Interactive Origami Simulation using GPU Computation. In Origami 7, Vol. 4. Tarquin, 1151-1166.

David A. Huffman. 1976. Curvature and creases: A primer on paper. IEEE Trans. Computers C-25 (1976), 1010-1019.

Ivan Izmestiev. 2017. Classification of flexible Kokotsakis polyhedra with quadrangular base. Int. Math. Res. Not. 3 (2017), 715-808.

Yannick L. Kergosien, Hironoba Gotoda, and Tosiyasu L. Kunii. 1994. Bending and Creasing Virtual Paper. Comput. Graph. Appl. 14 (1994), 40-48.

Martin Kilian, Simon Flöry, Zhonggui Chen, Niloy Mitra, Alla Sheffer, and Helmut Pottmann. 2008. Curved Folding. ACM Trans. Graph. 27, 3 (2008), \#75, 1-9.

Martin Kilian, Aron Monszpart, and Niloy J. Mitra. 2017. String Actuated Curved Folded Surfaces. ACM Trans. Graph. 36, 3 (2017), \#25, 1-13.

Antonios Kokotsakis. 1933. Über bewegliche Polyeder. Math. Ann. 107 (1933), 627-647. Mina Konaković, Keenan Crane, Bailin Deng, Sofien Bouaziz, Daniel Piker, and Mark Pauly. 2016. Beyond Developable: Computational Design and Fabrication with Auxetic Materials. ACM Trans. Graph. 35, 4 (2016), \#89, 1-11.

Mina Konaković-Luković, Pavle Konaković, and Mark Pauly. 2018. Computational Design of Deployable Auxetic Shells. In Advances in Architectural Geometry 2018, L. Hesselgren et al. (Eds.). Klein Publishing, 94-111.

Richard D. Koschitz. 2014. Computational design with curved creases: David Huffman's approach to paperfolding. Ph.D. Dissertation. MIT.
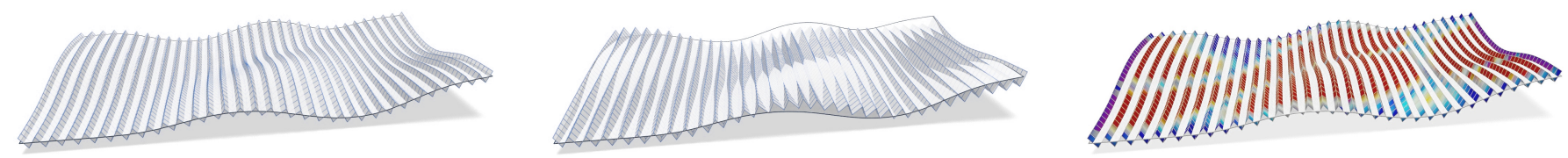

Fig. 25. Bad initialization. Here, evenly spaced curves are moved off a reference surface in direction of the normals to initialize folds. Subsequent optimization does not produce the desired result: In the center image, we enforce planarity of faces and developabilty, and end up with straight folds failing to approximate the reference surface. In the right hand image, we enforce proximity to the initial folds (with a larger value of $\lambda_{2}$ in the target functional) and fail to achieve planarity and developability. The color coding shows the deviation from developability. 

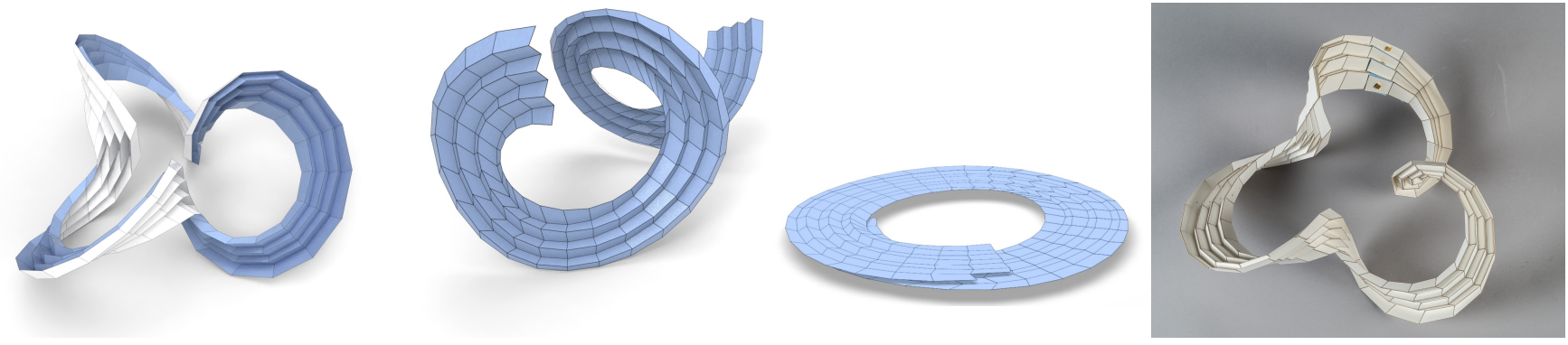

Fig. 26. Design of flexible mechanisms. This principal pleated structure was generated by evolution of pseudo-geodesics from a torus knot polyline. The principal property implies that it is a mechanism (after cutting to make it simply connected). We show the curved-crease surface, partial and complete unfolding, and a physical model. This model has been made by putting cardboard faces onto double adhesive film which is flexible but does not stretch. The $1 \mathrm{~mm}$ foil gap between faces acts as hinges. The local and also global assembly tolerances are about $0.2 \mathrm{~mm}$, compared to a centerline length of $1.25 \mathrm{~m}$. During flexion of the physical model, the constant fold angle is maintained (by visual inspection). We regard this example of mechanism design a main contribution of our paper.

Artur Lebée. 2015. From folds to structures, a review. Int. 7. Space Structures 30, 2 (2015), $55-74$.

Ting-Uei Lee, Xiaochen Yang, Jiayao Ma, Yan Chen, and Joseph M. Gattas. 2019. Elastic buckling shape control of thin-walled cylinder using pre-embedded curved-crease origami patterns. Int. 7. Mechanical Sciences 151 (2019), 322-330.

Yang Liu, Helmut Pottmann, Johannes Wallner, Yong-Liang Yang, and Wenping Wang. 2006. Geometric modeling with conical meshes and developable surfaces. ACM Trans. Graph. 25, 3 (2006), 681-689.

Jun Mitani. 2009a. A Design Method for 3D Origami Based on Rotational Sweep. Computer-Aided Des. \& Appl. 6 (2009), 69-79.

Jun Mitani. 2009b. Origami Sphere (crease patterns). available from http://mitani.cs. tsukuba.ac.jp (accessed August 2019).

Jun Mitani. 2012. Column-shaped origami design based on mirror reflections. F. Geom. Graphics 16 (2012), 185-194.

Jun Mitani and Takeo Igarashi. 2011. Interactive Design of Planar Curved Folding by Reflection. In Pacific Graphics, Short Papers. Eurographics, 77-81.

Shuhei Miyashita, Isabella DiDio, Ishwarya Ananthabhotla, Byoungkwon An, Cynthia Sung, Slava Arabagi, and Daniela Rus. 2015. Folding Angle Regulation by Curved Crease Design for Self-Assembling Origami Propellers. 7. Mechanisms Robotics 7 (2015), \#021013, 1-8.

Pierre-Olivier Mouthuy, Michael Coulombier, Thomas Pardoen, Jean-Pierre Raskin, and Alain M. Jonas. 2012. Overcurvature describes the buckling and folding of rings from curved origami to foldable tents. Nature Comm. 3 (2012), \#1290.

Klara Mundilova. 2019. On mathematical folding of curved crease origami: Sliding developables and parametrizations of folds into cylinders and cones. Computer-Aided Design 115 (2019), 34-41. Proc. SPM.

Helmut Pottmann, Qixing Huang, Bailin Deng, Alexander Schiftner, Martin Kilian, Leonidas Guibas, and Johannes Wallner. 2010. Geodesic Patterns. ACM Trans. Graph. 29, 4 (2010), \#43, 1-10.

Helmut Pottmann, Alexander Schiftner, Pengbo Bo, Heinz Schmiedhofer, Wenping Wang, Niccolo Baldassini, and Johannes Wallner. 2008. Freeform surfaces from single curved panels. ACM Trans. Graph. 27, 3 (2008), \#76, 1-10.

Michael Rabinovich, Tim Hoffmann, and Olga Sorkine-Hornung. 2018a. Discrete Geodesic Nets for Modeling Developable Surfaces. ACM Trans. Graph. 37, 2 (2018), 16:1-16:17.

Michael Rabinovich, Tim Hoffmann, and Olga Sorkine-Hornung. 2018b. The Shape Space of Discrete Orthogonal Geodesic Nets. ACM Trans. Graph. 37, 6 (2018), 228:1228:17.

Vlad A. Raducanu, Vasile D. Cojocaru, and Doina Raducanu. 2016. Structural Architectural Elements Made of Curved Folded Sheet Metal. In eCAADe 2016 - Complexity \& Simplicity, A. Herneoja et al. (Eds.). Vol. 2. Univ. Oulu, 409-416.

Otto Röschel. 2019. Curved Folding with Pairs of Cones. In Proceedings 18th Int. Conf. Geometry and Graphics, L. Cocchiarella (Ed.). Springer, 381-388.

Wolfgang Schief, Alexander Bobenko, and Tim Hoffmann. 2008. On the integrability of infinitesimal and finite deformations of polyhedral surfaces. In Discrete differential geometry, A. Bobenko et al. (Eds.). Oberwolfach Seminars, Vol. 38. Springer, 67-93.

Eike Schling, Denis Hitrec, and Rainer Barthel. 2018. Designing Grid Structures using Asymptotic Curve Networks. In Humanizing Digital Reality, K. de Rycke et al. (Eds.) Springer, 125-140. Proc. Design Modelling Symposium.

Keith A. Seffen. 2012. Compliant shell mechanisms. Phil. Trans. R. Soc. A 370 (2012), 2010-2026.

Justin Solomon, Etienne Vouga, Max Wardetzky, and Eitan Grinspun. 2012. Flexible developable surfaces. Comput. Graph. Forum 31 (2012), 1567-1576. Proc. SGP.
Keyao Song, Xiang Zhou, Shixi Zang, Hai Wang, and Zhong You. 2017. Design of rigidfoldable doubly curved origami tessellations based on trapezoidal crease patterns Proc. R. Soc. A 473 (2017), \#20170016, 1-18.

Oded Stein, Eitan Grinspun, and Keenan Crane. 2018. Developability of triangle meshes ACM Trans. Graph. 37, 4 (2018), \#77, 1-14.

Daniel M. Sussman, Yigil Cho, Toen Castle, Xingting Gong, Euiyeon Jung, Shu Yang, and Randall D. Kamien. 2015. Algorithmic lattice kirigami: A route to pluripotent materials. Proc. Nat. Ac. Sc. 112, 24 (2015), 7449-7453.

Tomohiro Tachi. 2009. Generalization of rigid foldable quadrilateral mesh origami. 7 . Int. Ass. Shell \& Spatial Structures 50 (2009), 173-179.

Tomohiro Tachi. 2010a. Geometric Considerations for the Design of Rigid Origami Structures. In Proc. IASS Symposium 2010. 771-782.

Tomohiro Tachi. 2010b. Origamizing polyhedral surfaces. IEEE Trans. Vis. Comp. Graphics 16, 2 (2010), 298-311

Tomohiro Tachi. 2013. Composite Rigid-Foldable Curved Origami Structure. In Proc. 1st Transformables Conf., F. Escrig and J. Sanchez (Eds.). Starbooks, Sevilla, 6 pp.

Tomohiro Tachi and Gregory Epps. 2011. Designing One-DOF Mechanisms for Architecture by Rationalizing Curved Folding. In Proc. ALGODE Symposium, Y. Ikeda (Ed.). Arch. Institute Japan, Tokyo, 14 pp. CD ROM.

Chengcheng Tang, Pengbo Bo, Johannes Wallner, and Helmut Pottmann. 2016. Interactive design of developable surfaces. ACM Trans. Graph. 35, 2 (2016), \#12, 1-12.

Sivan Toledo. 2003. TAucs, A Library of Sparse Linear Solvers. C library.

Zhiyan Wei, Zengcai Guo, Levi Dudte, Haiyi Liang, and L. Mahadevan. 2013. Geometric Mechanics of Periodic Pleated Origami. Phys. Rev. Lett. 110 (2013), \#215501, 1-5.

Walter Wunderlich. 1950a. Pseudogeodätische Linien auf Kegelflächen. Sitzungsber. II, Österr. Akad. Wiss. 158 (1950), 75-105.

Walter Wunderlich. 1950b. Pseudogeodätische Linien auf Zylinderflächen. Sitzungsber II, Österr. Akad. Wiss. 158 (1950), 61-73.

Walter Wunderlich. 1950c. Raumkurven, die pseudogeodätische Linien eines Zylinders und eines Kegels sind. Compos. Math. 8 (1950), 169-184.

Walter Wunderlich. 1950d. Raumkurven, die pseudogeodätische Linien zweier Kegel sind. Monatsh. Math. 54 (1950), 55-70.

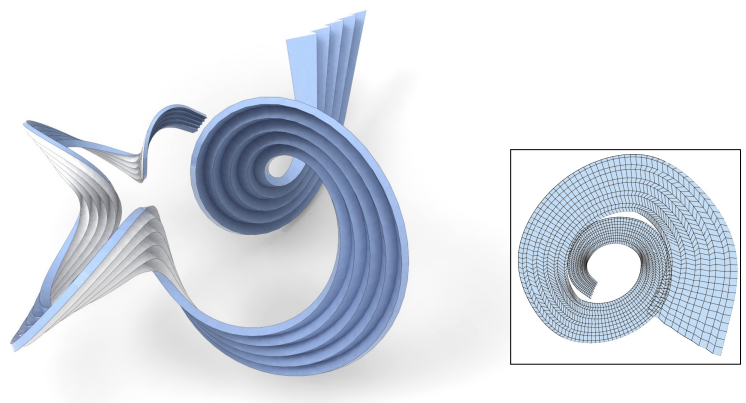

Fig. 27. Non-uniform evolution. This example of a principal PLS is based on non-uniform evolution of pseudo-geodesics from a given space curve, where the inclination angle remains constant, but the distance between curves is varying along the curve. The unfolding (see inset) is not 1-1. 